\title{
Numerical Investigation of a Shielded Chevron Nozzle
}

\author{
Brian C. Heberling* \\ Vantage Partners, NASA Glenn Research Center, Cleveland, Ohio, 44135
}

\begin{abstract}
Reynolds-Averaged Navier-Stokes simulations have been performed on a three-stream inverted velocity profile nozzle with and without various configurations of chevrons attached. The nozzle was mounted on a planform to imitate an engine mounted above a wing, shielding ground observers from engine noise. Several chevron designs intended to aggressively mix the jet and move noise sources upstream for shielding were examined to investigate their effects on noise and thrust. Numerical results for the baseline nozzle and one chevron configuration were compared with far-field noise and particle image velocimetry data obtained in NASA Glenn Research Center's Aero-Acoustic Propulsion Laboratory. A configuration in which chevrons alternate penetration into the primary stream and tertiary fan stream was explored using the Modern Design of Experiments approach. Short, high-penetration chevrons demonstrated a significant noise reduction for a relatively small thrust penalty.
\end{abstract}

\section{Introduction}

$\mathrm{T}$ HE Commercial Supersonic Technology project seeks to develop technologies that overcome the barriers to practical supersonic commercial aircraft. One of the major challenges to supersonic transports is reducing airport noise to acceptable levels. NASA's N+2 Supersonic Transport goals aim for a 10 to $20 \mathrm{~dB}$ effective perceived noise level (EPNL) cumulative decrease in airport noise below FAA Stage 3 noise standards ${ }^{1}$. Achieving this goal will require a large reduction in propulsion noise.

Lockheed Martin's N+2 concept vehicle, the LM1044, has been used by NASA as the basis for system studies of a supersonic transport. The LM1044 aircraft cruises at Mach 1.7 and carries 80 passengers with a 5000+ nautical mile range ${ }^{1}$. System analyses have indicated exhaust noise as the dominant noise source during airport operations, specifically at the lateral (sideline) certification point when noise is measured 450 meters from the runway centerline at the time of peak takeoff noise.

One promising strategy for reducing jet noise is the addition of chevrons to the nozzle. Chevrons increase streamwise vorticity, enhancing mixing within the plume for a small performance cost. The enhanced mixing decreases downstream low-frequency noise but often increases high-frequency noise close to the nozzle ${ }^{2}$. Thus, a designer must take care to balance the low-frequency noise reduction with the corresponding increase in high-frequency noise and drag in order to obtain a net benefit in effective perceived noise level (EPNL) for an acceptable performance penalty at cruise conditions. This balance has resulted in relatively low-penetration chevrons being used on flight hardware ${ }^{3}$.

Another strategy for jet noise reduction is the use of top-mounted propulsion to shield the engine from the ground observer. This has been shown to offer significant noise reduction from both the fan and the exhaust system ${ }^{4}$. Recent tests at NASA Glenn Research Center have indicated a 3-4 EPNdB benefit from top-mounted engine installation relative to an underwing configuration ${ }^{5}$. Combined with chevrons, top-mounted propulsion can shield the high-frequency sources close to the nozzle exit and lessen the penalty associated with enhanced mixing.

Technology advances in the area of Shape-Memory Alloys (SMAs) offer a method of reducing the other penalty associated with chevrons, the increase in drag. SMAs can be trained to "remember" a shape at certain temperatures, dependent upon the material properties. Turner et al ${ }^{6]}$ have tested chevrons with electrically-activated SMA strips through the center of the chevron, which can be actuated to bend each chevron into the exhaust. This technology allows for chevrons which aggressively penetrate into the flowpath during takeoff for a large noise reduction, yet flatten during cruise for a minimal thrust penalty.

With the need for decreased airport noise and the increasing potential to offset the penalties associated with chevrons, it is likely that chevron designs will trend towards more aggressive mixing. In this study, Reynolds-Averaged Navier-Stokes (RANS) computations were performed on an installed nozzle with various chevrons attached in order to investigate their impact on acoustic emissions and thrust. A jet noise prediction code was used to compute far-field noise from RANS solutions.

\footnotetext{
*Aerospace Engineer, Inlets \& Nozzles Branch, 21000 Brookpark Road, AIAA Member
} 
First, the design space was explored using chevrons of varying location, penetration, length, and number. Numerical results of the baseline nozzle and one chevron configuration are compared with particle image velocimetry (PIV) and far-field noise data obtained in the NASA Glenn Research Center's Aero-Acoustic Propulsion Laboratory (AAPL). Finally, the Modern Design of Experiments (MDOE) method is applied to explore a promising parameter space in which chevrons alternate penetration into the primary stream and fan stream.

\section{Geometry}

\section{A. Nozzle}

The nozzle being studied is a three-stream inverted velocity profile (IVP) plug nozzle ${ }^{7}$, shown in Figure 1 The exhaust system is designed for a variable cycle engine with two fan streams. The tip fan flow is split between the innermost ("inner") and outermost ("buffer") nozzle streams, while the core and inner fan flow is mixed internally and exhausted through the "primary" annulus surrounding the inner stream. The buffer stream only spans half of the nozzle circumference on the ground-facing side of the nozzle, while the inner and primary streams span the entire nozzle circumference. The IVP concept can potentially reduce jet noise relative to a traditional velocity profile for a supersonic jet ${ }^{8}$. Exhausting the high-speed primary stream through a thin annulus accelerates mixing relative to a typical exhaust configuration, reducing downstream low-frequency noise. The enhanced mixing increases high-frequency noise, but the buffer stream acts as a fluidic shield to mitigate this penalty, reducing the velocity gradient close to the nozzle exit. In this study only the take-off geometry configuration was examined. Tests and numerical studies were performed at modelscale. The primary stream exit diameter $D_{J}$ is approximately 6.7 inches.

\section{B. Test Planform}

The nozzle is installed on a pylon attached to a test planform and vertical tail (Figure 2), mimicking an engine mounted above the aft deck of the LM1044 concept aircraft. The pylon attaches to the outer lip of the buffer nozzle such that the buffer stream separates the other streams and the planform. The axial distance between the buffer stream lip and the pylon trailing edge is 28.3 inches or $4.2 D_{J}$. The pylon slopes away from the jet plume, with a radial distance of 3 inches or $0.4 D_{J}$ between the lip and trailing edge. Due to the location of the far-field microphone array on the ceiling of the test facility, tests were conducted with the model upside-down with the planform mounted above the nozzle. Numerical results are presented in the same orientation.

\section{Chevrons}

The nozzle has three possible chevron locations, each consisting of a $180^{\circ}$ arc: the primary nozzle on the freestream side, the primary nozzle on the buffer stream side, and the buffer nozzle. Several chevron designs will be examined in this work; described by their penetration angle relative to the engine centerline, axial length, and number of chevrons. Furthermore, several configurations of these chevrons are simulated with varying chevron designs at different locations.

\section{Design Space Exploration}

First, the baseline nozzle with no chevrons and a configuration with primary nozzle chevrons is simulated and compared with experimental data. Several other configurations are also simulated to examine how they compare with the experimentally tested chevrons. Each chevron design is described in Table 1 Chevrons are named based on which stream they are applied to; where PC and BC denote Primary Chevron and Buffer Chevron, respectively. The various chevron designs are applied to the nozzle in combinations to create a configuration defined by a three-digit code, with each digit corresponding to a chevron design described in Table 1 . The order of the digits are primary nozzle (freestream side), primary nozzle (buffer side), and buffer nozzle. 0 indicates no chevrons attached at that location. For example, configuration 240 designates the PC2 chevrons on the freestream side of the primary nozzle, the PC4 chevrons on the buffer stream side of the primary nozzle, and no chevrons on the buffer nozzle. 000 is the baseline configuration with no chevrons. Configurations 000, 240, 245, 270, and 080 are simulated, with test data available for the first two. Side views of each of these configurations can be seen in Figure 3.

Note that the attachment of the pylon to the buffer nozzle lip leaves room for only 6 BC5 chevrons. The PC7 chevrons are similar to the PC4 chevrons with a significant difference: every other chevron penetrates into the buffer stream rather than the primary stream. Due to the even number of chevrons, an asymmetry is present. An observer 
looking upstream into the nozzle would see the chevron on the left end of the PC7 strip pointing inward and the chevron on the right end pointing outward.

Table 1. Descriptions of each chevron design.

\begin{tabular}{|l|c|c|c|c|}
\hline Name & Penetration $\left(^{\circ}\right)$ & Length (in) & Count & Location \\
\hline PC2 & 16 & 0.625 & 8 & Primary (Freestream) \\
\hline PC4 & 16 & 0.625 & 8 & Primary (Buffer) \\
\hline BC5 & 10 & 1.000 & 6 & Buffer \\
\hline PC7 & $16 /-16$ & 0.625 & 8 & Primary (Buffer) \\
\hline PC8 & 22 & 0.850 & 16 & Primary (Freestream) \\
\hline
\end{tabular}

\section{Modern Design of Experiments Matrix}

Initial design space exploration results indicate significant benefits to acoustic emissions and a relatively small thrust penalty from the 270 configuration with alternating penetration chevrons. To explore this concept further, a matrix of alternating penetration chevron configurations was designed using the Modern Design of Experiments (MDOE) method ${ }^{9}$. Each configuration consists of nine chevrons attached on the buffer side of the primary nozzle in which the two end chevrons penetrate into the primary stream. Three parameters are defined: penetration into the primary stream, penetration into the buffer stream, and axial length. For each chevron configuration, inward- and outward-pointing chevrons have the same axial length. The design matrix is displayed in Table 2 along with the nomenclature to describe the different chevron designs. The PC2 chevrons are attached on the freestream side of the primary nozzle and do not vary between MDOE configurations. No chevrons are located on the buffer stream lip.

In a three-dimensional parameter study such as this, the MDOE analysis involves the eight corners of the parameter space being simulated along with one center point. The center is used to evaluate the linear model produced by the corners and identify the existence of curvature. If little or no curvature is present, the linear model can be used to make predictions inside the parameter space. A diagram of the parameter space as well as each chevron configuration is displayed in Figure 4

Table 2. Modern Design of Experiments matrix.

\begin{tabular}{|l|c|c|c|}
\hline Parameter & Low Level & High Level & Center \\
\hline Penetration (Primary) $\left(^{\circ}\right)$ & 10 & 22 & 16 \\
\hline Penetration (Buffer) $\left(^{\circ}\right)$ & 10 & 22 & 16 \\
\hline Length (in) & 0.400 & 0.850 & 0.625 \\
\hline Nomenclature & P10B10L40 & P22B22L85 & P16B16L63 \\
\hline
\end{tabular}

\section{Methodology}

\section{A. Solver}

Simulations were performed using FUN3D ${ }^{10}$ version 12.7, an unstructured flow solver developed at the NASA Langley Research Center. FUN3D is a node-based, finite volume RANS solver. The two-equation Menter Shear Stress Transport (SST) turbulence model ${ }^{[11}$ was used and integrated to the wall. All calculations were steady state.

Each simulation was run at conditions representative of a supersonic transport flying at the FAA sideline noise certification point, at which the noise after takeoff is at a maximum. Engine cycle conditions were obtained from system studies ${ }^{1}$. Nozzle boundary conditions are presented in Table 3 . The nozzle pressure ratio (NPR) is the ratio of the stream's stagnation pressure to the ambient pressure, and the nozzle temperature ratio (NTR) is the ratio of the stream's 
stagnation temperature to the ambient temperature. The freestream Mach number was 0.3 , the ambient pressure was $14.7 \mathrm{psia}$, and the ambient temperature was $519^{\circ} \mathrm{R}$.

Table 3. Approximate nozzle boundary conditions.

\begin{tabular}{|l|c|c|}
\hline Stream & NPR & NTR \\
\hline Inner & 1.8 & 1.3 \\
\hline Primary & 2.0 & 1.9 \\
\hline Buffer & 1.8 & 1.3 \\
\hline
\end{tabular}

\section{B. Convergence}

Calculations were performed on NASA's Pleiades supercomputer. Each simulation used 1180 Ivy Bridge processor cores to run for 90,000 iterations, ramping the global CFL number from 0.5 to 10.0. The wall clock time of each simulation was approximately 45 hours. Residuals of the RANS equations and turbulence model were monitored for convergence, as well as surface forces and mass flow rates for each nozzle stream. The flowfield was also observed over the final 10,000 iterations to ensure no change.

\section{Noise Predictions}

Noise predictions were obtained using $\mathrm{mSrc}$ version 4.2. $\mathrm{mSrc}$ is a simple acoustic analogy code used for calculating jet noise from a point cloud, provided by FUN3D for each CFD solution. mSrc relates source strength to turbulent kinetic energy and source frequency to turbulent timescales. $\mathrm{mSrc}$ is useful for predicting differences in far-field noise due to geometry changes or for analyzing noise source locations. By first applying $\mathrm{mSrc}$ to the baseline nozzle with no chevrons, the effects of the different chevron designs can be evaluated on a delta basis. mSrc also includes a module for analyzing installation effects, which is used for each calculation to be discussed. Maekawa's barrier theory is applied to calculate shielding and reflection from the test planform.

\section{Grids}

Hybrid grids for each geometry were generated using Pointwise version 18.0 R4. In order to accurately resolve the jet plume, a large amount of grid must be concentrated within the mixing layer. Based on the recommendations of Dippold $\frac{12}{2}$, structured-like hexahedra blocks are used to model the plume and unstructured blocks are used elsewhere.

Each grid consists of approximately 48 million nodes. The structured-like plume blocks remained the same through each grid and included 30 million nodes. Figure 5 provides a view of the full grid domain. The upstream far-field diameter was $25 D_{J}$, expanding to $40 D_{J}$ downstream. The axial length of the grid domain was $85 D_{J}$, ending $65 D_{J}$ downstream of the primary nozzle exit. The plume blocks extend from one inch downstream of the primary nozzle exit to $35 \mathrm{D}_{\mathrm{J}}$ downstream. Figure 6 shows cross-sectional views of the plume blocks. Because of the nozzle plug, the first part of the plume grid is an annulus. Viscous wall spacing on surfaces were set to hold y+ close to unity.

A grid convergence study was performed on the 240 and 270 chevron configurations. Refined grids of 61 million nodes were generated by increasing the number of streamwise and radial nodes within the structured-like plume blocks. An examination of nozzle forces, flowfields, and power spectral density (PSD) as reported by mSrc showed a negligible difference between the refined grids and the baseline grids of 48 million nodes.

\section{E. Experimental Testing}

Experimental testing of the installed nozzle took place in NASA Glenn Research Center's Aero-Acoustic Propulsion Laboratory (AAPL), a geodesic dome with acoustically treated walls. AAPL uses the Nozzle Acoustic Test Rig (NATR $)^{\sqrt{13}}$ to simulate flight conditions with a 53 inch diameter free jet which exits a few feet upstream of the nozzle. Further description of the AAPL facility and discussions of test results including far-field noise ${ }^{\frac{14}{4}}$, PIV data $\frac{15}{15}$, and phased array measurements ${ }^{16}$ can be found in companion papers. Previous simulations have shown a negligible difference in results between modeling the Mach 0.3 free jet with a surrounding quiescent flow; or ignoring the cylindrical NATR free jet and setting a uniform freestream Mach number of 0.3 with no surrounding quiescent flow. For simplicity, NATR was not modeled. 


\section{Results}

This section examines RANS simulations at sideline flight conditions of the installed three-stream IVP nozzle with and without chevrons attached. Simulations were peformed with FUN3D and postprocessed using the mSrc acoustic analogy code. First, numerical results are compared with wind tunnel data for the baseline nozzle and one chevron configuration. Next, the design space is explored by investigating chevrons of varying location, length, number, and penetration. Finally, the Modern Design of Experiments method is used to further analyze the alternating penetration chevron concept.

\section{A. Design Space Exploration}

\section{Comparison of CFD and Test Data}

Far-field noise and PIV test data is available for two configurations: 000, the baseline nozzle with no chevrons; and 240, with chevrons attached on both sides of the primary nozzle. Far-field noise will be examined at a 1-foot, lossless condition and a polar angle of $90^{\circ}$, placing the observer on the opposite side of the test planform from the jet, or on the ground underneath the conceptual aircraft. Figure 7 compares the power spectral density (PSD) between AAPL data and CFD. On an absolute basis, mSrc predictions compare reasonably well with test data. Numerical results are underpredicting the low- and high-frequency noise and overpredicting PSD at mid-range frequencies. Over the majority of the frequency range, CFD is within $2 \mathrm{~dB}$ of the test data. However, at high frequencies CFD underpredicts noise for the baseline nozzle by nearly $6 \mathrm{~dB}$. AAPL tests indicated an increase in low-frequency noise at upstream and broadside angles ${ }^{14}$ to the jet axis, corresponding with a trailing edge dipole caused by the turbulent jet interacting with the trailing edge of the test planform. As mSrc is only intended to predict jet noise and has no functionality for trailing edge noise, the noise predictions from CFD will not include the trailing edge dipole. This at least partially explains the discrepancies at low frequencies for both configurations.

The delta PSD plot shows the difference in PSD between the baseline nozzle and 240 chevron configuration at each frequency, where a positive value indicates a benefit to noise from chevrons. The numerical results demonstrate a benefit from chevrons over a larger frequency range than observed in the experimental data. The high-frequency crossover, or the frequency at which chevrons begin to provide a penalty rather than a benefit to noise, occurs at $20 \mathrm{kHz}$ according to $\mathrm{mSrc}$ but at $10 \mathrm{kHz}$ in the test data. However, the numerical results indicate a much larger penalty from chevrons at high frequencies. The CFD predicts a $7 \mathrm{~dB}$ increase in PSD at $100 \mathrm{kHz}$ from chevrons, while AAPL data shows that the chevron penalty is largest at $20 \mathrm{kHz}$ and is less than $3 \mathrm{~dB}$. On a delta basis, CFD and mSrc together provide good estimates of low-frequency noise, but overpredict both the benefit from chevrons at mid-range frequencies and the penalty at high frequencies.

A comparison of axial velocity between CFD and PIV data at eight stations for both of these configurations is presented in Figure 8 . The PIV contours are blanked where the data quality metric ${ }^{17}$ is less than 0.8 . This is most apparent at the upstream PIV stations where the nozzle plug and test planform hinder data acquisition. Overall, there is good agreement between the CFD and PIV data. The CFD indicates slightly higher velocites in the baseline nozzle plume, most noticeably downstream of the plug $\left(\mathrm{x} / \mathrm{D}_{\mathrm{J}}=1.5\right)$ and near the end of the potential core $\left(\mathrm{x} / \mathrm{D}_{\mathrm{J}}=5.0,7.6\right)$.

This is also the case for the chevron configuration, albeit to a lesser extent. Here, the difference in plume velocities is most apparent at $x / D_{J}=5.0$. Closer to the nozzle, there is excellent agreement in both the shape and magnitude of the axial velocity contours. However, small features such as the high velocity tendrils downstream of the chevron valleys are far more apparent in the CFD. Note the small high-velocity regions on both sides of the nozzle, between the upper and lower chevron strips. In the upstream axial stations, these areas are more defined in the CFD solution which shows slightly higher velocities. Downstream contours show that these regions are important to the development of the non-axisymmetric plume shape. The PIV demonstrates that these features migrate upwards, morphing the plume into a shield shape. In the CFD, these features are mixed more quickly such that the plume appears mostly round by station 7 .

These same axial stations are examined in regards to turbulent kinetic energy in Figure 9. Both CFD solutions are overpredicting turbulence throughout the plume compared with the PIV data. There is good agreement in the shape of the turbulence, but a large discrepancy in magnitudes. Both the CFD and PIV show the turbulence extending from the plume to the pylon, leading to the trailing edge dipole. Downstream of the baseline nozzle, the CFD displays higher turbulence levels on the bottom of the nozzle in the mixing layer between the primary stream and freestream air. The PIV data indicates a more axisymmetric distribution of turbulence, although downstream $\left(\mathrm{x} / \mathrm{D}_{\mathrm{J}}=3.8,5.0\right)$ there does appear to be slightly higher turbulence on the bottom of the plume. 
The 240 CFD shows a large increase in turbulent kinetic energy close to the nozzle resulting from the chevrons. Although downstream of the chevrons the PIV data shows similar features as the CFD, the increase in mixing is not nearly as great. This is consistent with the PSD plot discussed earlier, where the numerical results predicted a much larger high-frequency penalty from the addition of chevrons than the test data showed. The regions on either side of the nozzle leading to the downstream shield shape of the plume are again apparent in the turbulence contours.

Although there is disagreement between the CFD and PIV data in the magnitude of the turbulence in the plume, both data sets display the same trend resulting from chevrons. The addition of chevrons to the nozzle increases mixing close to the nozzle exit. The increase in small-scale turbulent mixing causes the increase in high-frequency noise exhibited by Figure 7 The enhanced mixing close to the nozzle results in decreased mixing downstream in the plume where larger-scale structures dominate the jet, decreasing low-frequency noise.

\section{Chevron Nozzle CFD Comparison}

Numerical results for each chevron configuration will now be compared to evaluate the benefits and penalties of each configuration. The various chevron designs are described in Table 1 The 245 and 270 configurations are both variations of the 240 chevron configuration. In the former; six large, low-penetration chevrons are added to the buffer stream to examine whether they would interact with the downstream primary chevrons. In the latter, every other chevron on the buffer stream, or planform, side of the primary nozzle has been flipped such that the penetration is into the buffer stream rather than the primary stream. The intent was to reduce drag due to the lower-velocity fan air. Finally, the 080 configuration consists of sixteen long, thin, high-penetration chevrons on the freestream side of the primary nozzle.

Figure 10 presents the power spectral density as computed by $\mathrm{mSrc}$ for each simulation, relative to the baseline nozzle without chevrons. Positive values indicate a benefit from chevrons and negative values designate a penalty. Each geometry provides a reduction to low-frequency noise and an increase at high-frequencies. There is a negligible difference in PSD for the 240 and 245 chevrons. The alternating penetration 270 chevrons provide the largest noise reduction. This configuration is as much as $2 \mathrm{~dB}$ quieter than 240 at $1 \mathrm{kHz}$. Surprisingly, there is no increase in high-frequency noise relative to 240 . The 080 configuration provides the least noise benefit of all, but this corresponds with the smallest high-frequency penalty. As chevrons are only present on one side of the nozzle, the small amount of noise reduction is not a surprise.

Overall sound pressure level (OASPL) at $90^{\circ}$ and thrust for each chevron configuration are compared in Table 4. The 245 chevrons provided the same noise reduction as 240 but increased drag. 080 also performed worse than 240 , with a lower OASPL benefit but larger thrust penalty. However, the alternating penetration 270 chevrons provided the largest noise reduction as well as the smallest thrust penalty.

Table 4. CFD predictions for OASPL benefit and thrust penalty relative to the baseline nozzle (000).

\begin{tabular}{|l|c|c|}
\hline Configuration & ${\text { OASPL Benefit at } \mathbf{9 0}^{\circ}}[\mathbf{d B}]$ & Thrust Penalty $^{-}$ \\
\hline 240 & 3.0 & $4.7 \%$ \\
\hline 245 & 3.0 & $5.4 \%$ \\
\hline 270 & 3.5 & $4.5 \%$ \\
\hline 080 & 2.6 & $5.0 \%$ \\
\hline
\end{tabular}

Next, the flowfield downstream of each chevron configuration will be examined with cross-stream turbulent kinetic energy contours (Figure 11]. As expected from the PSD plot, there is very little difference in the mixing downstream of the 240 and 245 chevrons. 245 exhibits slightly higher turbulence at the first axial station on the upper half of the plume, downstream of the buffer chevrons.

Contrarily, the alternating penetration 270 chevrons have a large effect on the flowfield on the top of the plume. Both the 240 and 270 chevrons exhibit greatly enhanced mixing and high peaks of turbulent kinetic energy in the first two axial stations. However, the alternating penetration appears to extend this mixing downstream such that high turbulence levels continue to the 3rd and 4th axial stations.

Downstream, the plume is similar to that of the 240 configuration but with decreased turbulence consistent with the reduced low-frequency noise. The shape is less uniform circumferentially, and the plume has been deflected slightly upwards. The high-turbulence regions on either side of the nozzle leading to the shield shape are still present. However, an examination of the plume downstream of the planform shows higher turbulence levels on the right side of the nozzle 
than the left side. Recall that the 270 configuration is asymmetric, with the chevron on the left end penetrating inward and the chevron on the right end penetrating outward. The turbulence peaks on the right side of the nozzle, seen at stations 5 and 6, indicate that it may be desirable for the end chevrons to penetrate inwards. For this reason, the alternating penetration chevron designs discussed in the next section are symmetric with the end chevrons penetrating into the primary stream. It should also be noted that this configuration increases turbulence near the trailing edge of the pylon and planform, although it is not clear whether this would increase or decrease noise associated with the trailing edge dipole.

The 080 chevrons exhibit mild enhanced mixing, compared with the other configurations. There are high levels of turbulence only at $\mathrm{x} / \mathrm{D}_{\mathrm{J}}=0.4$, and significantly decreased turbulence in the next few stations. Downstream of the planform, turbulence levels are much higher than shown by the other geometries yet still lower than those shown by the baseline nozzle. This is consistent with the PSD plot discussed earlier, which showed that the 080 chevrons provide both a smaller low-frequency benefit and smaller high-frequency penalty relative to the other configurations.

Cross-stream plots of axial vorticity for each configuration are presented in Figure 12. There is a negligible difference between the 240 and 245 vorticity fields, consistent with the turbulent kinetic energy contours. The flowfield downstream of the 270 chevrons highlights the differences in mixing with the 240 configuration. Downstream of each chevron, two vortices of opposite sign are observed. The alternating penetration sets up pairs of co-rotating vortices rather than the counter-rotating vortices typical of chevrons. The subsequent merging of the neighboring co-rotating vortices results in stronger vorticity and intensified mixing. Weak vortices are seen downstream of the 080 chevrons at $0.4 \mathrm{D}_{\mathrm{J}}$ but they quickly dissipate, consistent with the mild enhanced mixing seen in the turbulence flowfield.

These results indicate that alternating penetration is a concept worth exploring further. The next section will examine alternating penetration chevrons using the Modern Design of Experiments method.

\section{B. MDOE Results}

Nine RANS simulations of alternating penetration chevrons were performed based on the MDOE matrix described in Table 2: the eight corners and center of the parameter space. The results of these calculations will be examined to investigate each chevron configuration's effects on acoustic emissions and thrust.

PSD predictions at $90^{\circ}$ as calculated by $\mathrm{mSrc}$ are presented in Figure 13 , relative to the base nozzle with no chevrons. Positive numbers designate a benefit from chevrons and negative numbers indicate a noise penalty. The 240 and 270 chevron configurations are also included for comparison. At mid-range frequencies, the MDOE chevrons provide as much as a $6 \mathrm{~dB}$ reduction in PSD while at high frequencies the penalty is roughly $7 \mathrm{~dB}$. There is little deviation between chevrons at high frequencies, for each configuration the high-frequency crossover is near $15 \mathrm{kHz}$. Noise reduction varies more at low- to mid-range frequencies, where the benefit varies as much as $2 \mathrm{~dB}$ between the "best" and "worst" configurations. Only one configuration (P22B22L85) appears to be significantly better than the 270 chevrons in terms of PSD.

Reduction in broadside OASPL and thrust loss is compared in Figure 14 for each chevron calculation. OASPL benefit ranges from 2.6 to $3.8 \mathrm{~dB}$, while values of thrust loss are between $3.4 \%$ and 5.5\%. In general, the MDOE chevrons exhibit superior performance to the other configurations. For a given reduction in noise, an alternating penetration configuration had a smaller performance penalty. For example, the 240 chevrons reduced OASPL by approximately 3.0 $\mathrm{dB}$ for a $4.7 \%$ thrust penalty. The P10B22L40 chevrons provided a similar benefit to noise for only a $3.6 \%$ thrust penalty.

A linear regression analysis using OASPL at $90^{\circ}$ and thrust as the dependent variables outputs the MDOE coefficients presented in Table 5. Recall that the center point of the parameter space was simulated to evaluate the system for curvature. Table 6 compares the numerical results with the predictions obtained from the linear MDOE model. The minimal difference between CFD and MDOE prediction indicates a lack of curvature in the system, meaning that the linear model should provide accurate predictions for OASPL and thrust inside the defined parameter space.

The relative magnitudes of each MDOE coefficient can be analyzed to investigate each parameter's impact on OASPL and thrust. For both dependent variables, length has the most significant effect. As length is the only parameter to affect all chevrons on the planform side of the primary stream, while the two penetration parameters only affect four or five chevrons, this is not a surprise. The buffer penetration of the outward-pointing chevrons provides both a larger benefit to noise and a smaller penalty to thrust than the primary penetration parameter.

The interactions between primary and buffer penetration are insignificant for both noise and thrust. However, the interactions between the length parameter and either penetration parameter have an adverse effect on thrust due to the increased blockage and wetted area.

An optimizer was used with the MDOE model to maximize the ratio of OASPL reduction in $\mathrm{dB}$ to percent thrust 
loss. When constrained to the parameter space, the optimizer outputs the already-simulated P10B22L40 configuration with a ratio of 0.83 . For reference, the "worst" MDOE configuration by this metric, P22B22L85, has a ratio of 0.68 and the 240 chevrons provide a ratio of 0.65 .

To examine whether the MDOE model would remain accurate outside of the parameter space, the optimizer was then used with an enlarged parameter space. The penetration dimensions were doubled, opening the optimizer to values up to $28^{\circ}$; while chevron length was constrained to between 0.2875 and 0.9625 inches. The optimizer again suggested a configuration with high-penetration, short chevrons denoted as P28B28L29; which was then simulated using FUN3D. The OASPL reduction and thrust loss associated with this configuration is given in Figure 14. The linear model predicted an OASPL benefit to thrust loss ratio of 0.91 , but the numerical results calculated a ratio of 0.82 , indicating that the MDOE model is not valid outside of the parameter space.

Table 5. MDOE coefficients provided by linear regression. Parameters are normalized to $-1 \leq P, B, L \leq 1$.

\begin{tabular}{|c|c|c|c|c|c|c|c|}
\hline & & Primary Penetration & Buffer Penetration & Length & \multicolumn{3}{|c|}{ Parameter Interactions } \\
\hline & Intercept & $\mathrm{P}$ & B & $\mathrm{L}$ & $\mathrm{P} * \mathrm{~B}$ & $\mathrm{P} * \mathrm{~L}$ & $\mathrm{~B} * \mathrm{~L}$ \\
\hline OASPL [dB] & 94.2 & -0.10 & -0.16 & -0.19 & 0.001 & 0.005 & -0.054 \\
\hline Thrust [lbf] & 487.2 & -1.58 & -1.07 & -2.51 & -0.036 & -0.792 & -0.575 \\
\hline \multicolumn{8}{|c|}{ OASPL $[\mathrm{dB}]=94.2-0.10 * \mathrm{P}-0.16 * \mathrm{~B}-0.19 * \mathrm{~L}+0.001 * \mathrm{P} * \mathrm{~B}+0.005 * \mathrm{P} * \mathrm{~L}-0.054 * \mathrm{~B} * \mathrm{~L}$} \\
\hline \multicolumn{8}{|c|}{ Thrust $[\mathrm{lbf}]=487.2-1.58 * \mathrm{P}-1.07 * \mathrm{~B}-2.51 * \mathrm{~L}-0.036 * \mathrm{P} * \mathrm{~B}-0.792 * \mathrm{P} * \mathrm{~L}-0.575 * \mathrm{~B} * \mathrm{~L}$} \\
\hline
\end{tabular}

Table 6. Curvature assessment of the MDOE parameter space.

\begin{tabular}{|l|r|r|r|}
\hline & CFD (P16B16L63) & Linear Prediction & \% Difference \\
\hline OASPL $[\mathrm{dB}]$ & 94.1 & 94.2 & $0.09 \%$ \\
\hline Thrust $[\mathrm{lbf}]$ & 487.5 & 487.2 & $-0.07 \%$ \\
\hline
\end{tabular}

\section{Summary and Conclusions}

A three-stream inverted velocity profile nozzle has been simulated with varying configurations of attached chevrons. The nozzle was installed on a planform imitiating the aft deck of an aircraft, shielding ground observers from engine noise. Far-field noise was calculated for each resulting flowfield using a jet noise prediction code. Comparisons with PIV data for the baseline nozzle and the 240 chevron configuration indicate excellent agreement in the meanflow, but the RANS calculations show higher levels of turbulent kinetic energy in the mixing layer. Despite the discrepancy in plume turbulence, far-field noise predictions and test data compare reasonably well, with predictions within $2 \mathrm{~dB}$ for the majority of the frequency range. On a delta basis, CFD provides a good estimate for the difference in low-frequency noise between the chevron and baseline configuration. However, both the benefit from chevrons at mid-range frequencies and the penalty at high frequencies is overestimated.

The design space was first explored by investigating chevron configurations of differing location, number, penetration, and length. Chevrons that alternate penetration into the primary stream and tertiary buffer stream demonstrate a large reduction in low-frequency noise for relatively small high-frequency and thrust penalties, compared with the non-alternating chevrons. To explore the concept further, the Modern Design of Experiments method was used to develop a three-parameter matrix of alternating penetration chevrons varying primary stream penetration, buffer stream penetration, and axial length.

RANS results indicate that the alternating penetration chevrons provide more noise reduction for a lower thrust penalty, relative to the experimentally tested 240 chevrons. The MDOE analysis provides linear models for broadside OASPL and thrust, which suggest short, high-penetration chevrons to minimize noise while maximizing thrust. The linear models were sufficient for predictions inside the parameter space where the system demonstrated no curvature, but a calculation outside of the parameter space did not agree with predictions. 
Numerical calculations have demonstrated that chevrons with alternating penetration offer a promising reduction in noise for a smaller thrust penalty than a similar non-alternating configuration. This study only examined the performance at sideline flight conditions, but future work could investigate noise at the take-off or approach noise certification points. Performance at cruise conditions should also be examined, potentially with shape memory alloy-actuated chevrons.

\section{Acknowledgments}

This work was supported by NASA's Commercial Supersonic Technology Project under the Advanced Air Vehicles Program. Computational resources supporting this work were provided by NASA's High-End Computing Program through the NASA Advanced Supercomputing Division at Ames Research Center. The author is grateful to James Bridges, Vance Dippold, and Charles Trefny for their editorial help. Additional thanks are due to James Bridges for the use of his jet noise prediction code mSrc.

\section{References}

[1] Morgenstern, J., Buonanno, M., Yao, J., et al., “Advanced Concept Studies for Supersonic Commercial Transports Entering Service in the 2018-2020 Period Phase 2," NASA/CR - 2015-218719, July 2015.

[2] Henderson, B., and Bridges, J., “An MDOE Investigation of Chevrons for Supersonic Jet Noise Reduction," 16th AIAA/CEAS Aeroacoustics Conference, 2010.

[3] Zaman, K., Bridges, J. E., and Huff, D., "Evolution from 'Tabs' to 'Chevron Technology' - A Review," International Journal of Aeroacoustics, Vol. 10, No. 5-6, 2011, pp. 685-709.

[4] Bridges, J., Podboy, G. G., and Brown, C. A., "Testing Installed Propulsion for Shielded Exhaust Configurations," 22nd AIAA/CEAS Aeroacoustics Conference, 2016.

[5] Bridges, J., “Aeroacoustic Validation of Installed Low Noise Propulsion for NASA's N+2 Supersonic Airliner," 2018 AIAA Aerospace Sciences Meeting, 2018.

[6] Turner, T. L., Cabell, R. H., Cano, R. J., and Fleming, G. A., "Design, Fabrication, and Testing of a SMA Hybrid Composite Jet Engine Chevron,” Smart Structures and Materials 2006: Smart Structures and Integrated Systems, 2006.

[7] Bridges, J., Brown, C. A., and Seidel, J. A., "NASA's Pursuit of Low-Noise Propulsion for Low-Boom Commercial Supersonic Vehicles," 2018 AIAA Aerospace Sciences Meeting, 2018.

[8] Henderson, B., Bridges, J., and Wernet, M. P., "Jet Noise Reduction Potential From Emerging Variable Cycle Technologies," NASA/TM - 2012-217744, Nov. 2012.

[9] Deloach, R., "The Modern Design of Experiments: A Technical and Marketing Framework," 21st AIAA Advanced Measurement Technology and Ground Testing Conference, 2000.

[10] Biedron, R. T., Carlson, J.-R., Derlaga, J. M., et al., “FUN3D Manual: 12.7,” NASA/TM - 2015-218761, May 2015.

[11] Menter, F. R., “Two-Equation Eddy-Viscosity Turbulence Models for Engineering Applications,” AIAA Journal, Vol. 32, No. 8, 1994, pp. 1598-1605.

[12] Dippold, V. F., “Generating a Grid for Unstructured RANS Simulations of Jet Flows,” 2018 Fluid Dynamics Conference, 2018.

[13] Castner, R. S., “The Nozzle Acoustic Test Rig: An Acoustic and Aerodynamic Free-Jet Facility,” NASA/TM - 1995-106495, Apr. 1994.

[14] Bridges, J., "Noise Measurements of a Low-Noise Top-Mounted Propulsion Installation for a Supersonic Airliner," AIAA SciTech Forum 2019, 2019.

[15] Bridges, J., and Wernet, M. P., "PIV Measurements of a Low-Noise Top-Mounted Propulsion Installation for a Supersonic Airliner," AIAA SciTech Forum 2019, 2019.

[16] Cluts, J., Bridges, J., and Wernet, M. P., "Sound Source Maps of a Low-Noise Top-Mounted Propulsion Installation for a Supersonic Airliner,” AIAA SciTech Forum 2019, 2019.

[17] Bridges, J., and Wernet, M. P., “The NASA Subsonic Jet Particle Image Velocimetry (PIV) Dataset,” NASA/TM - 2011-216807, Nov. 2011. 


\section{Figures}

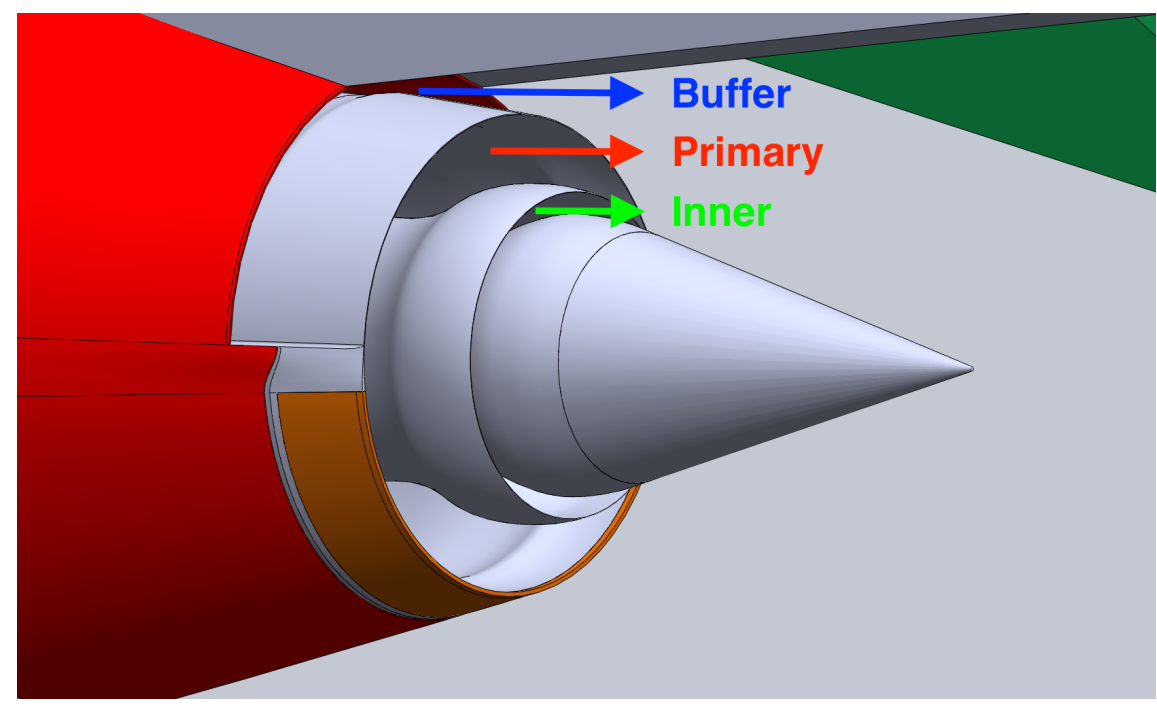

Figure 1. The three-stream inverted velocity profile nozzle used in these simulations ( 000 baseline configuration).
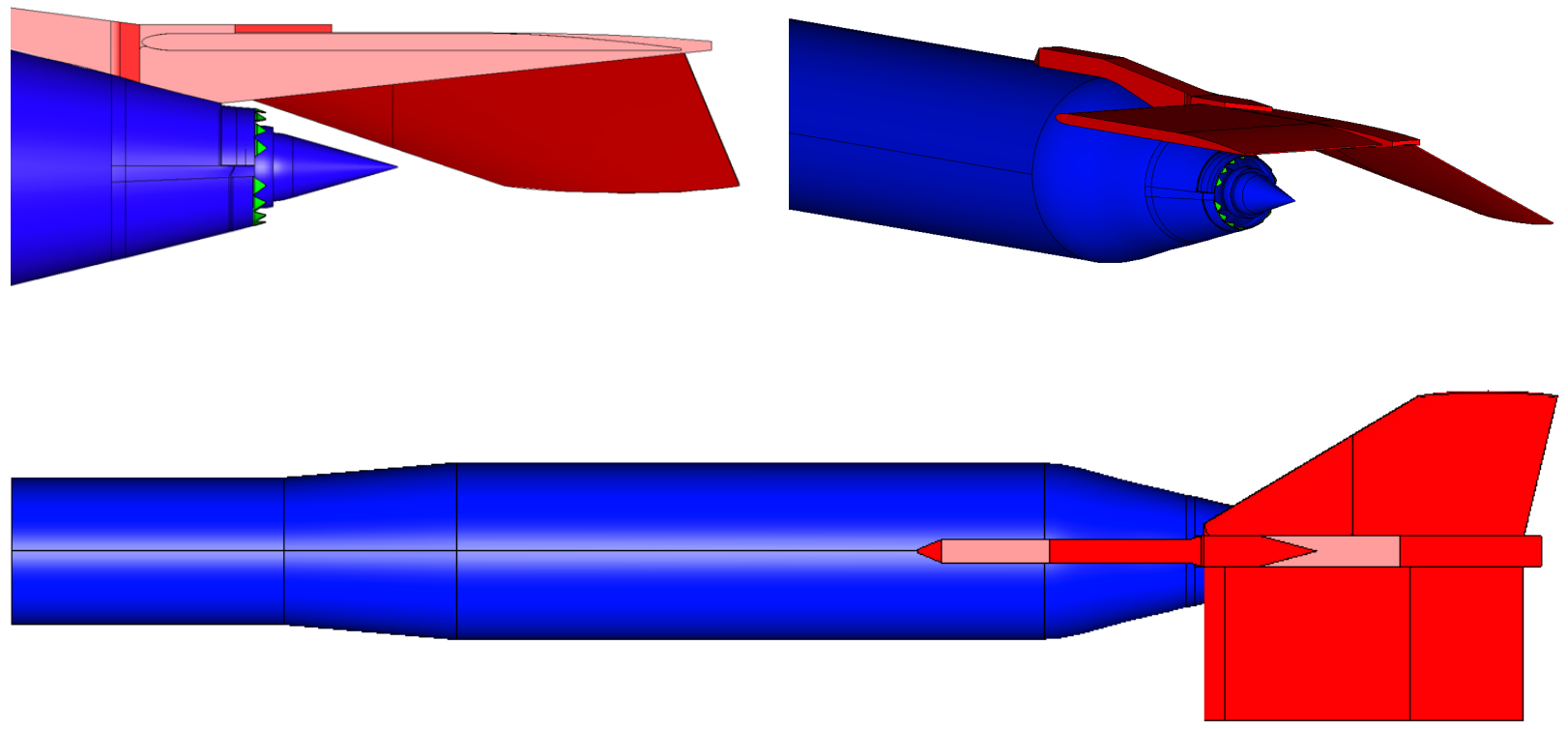

Figure 2. Views of the test planform meant to imitate the aft deck of the LM1044 concept vehicle (240 chevron configuration). 


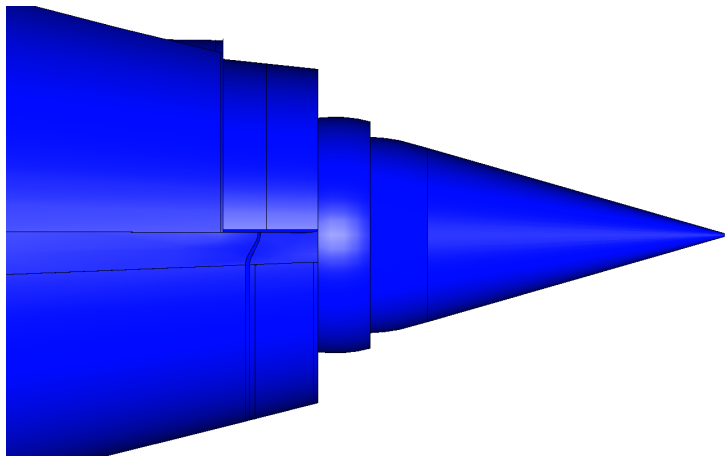

(a) 000 Configuration

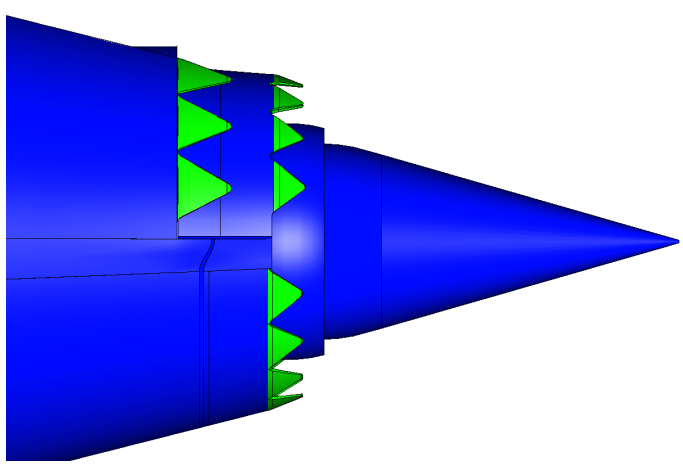

(c) 245 Configuration

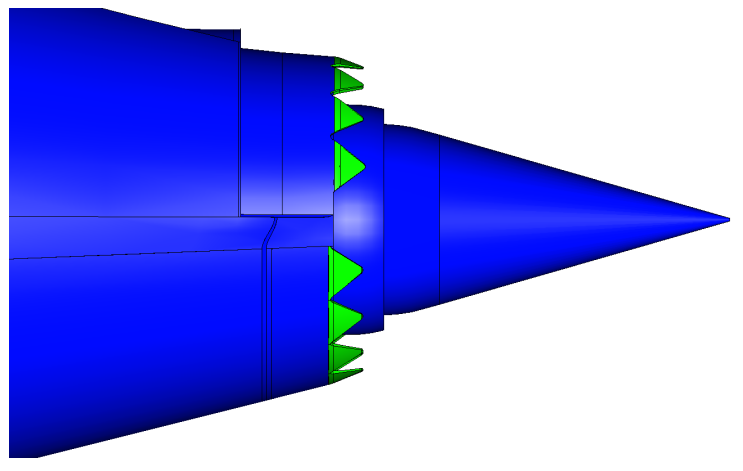

(b) 240 Configuration

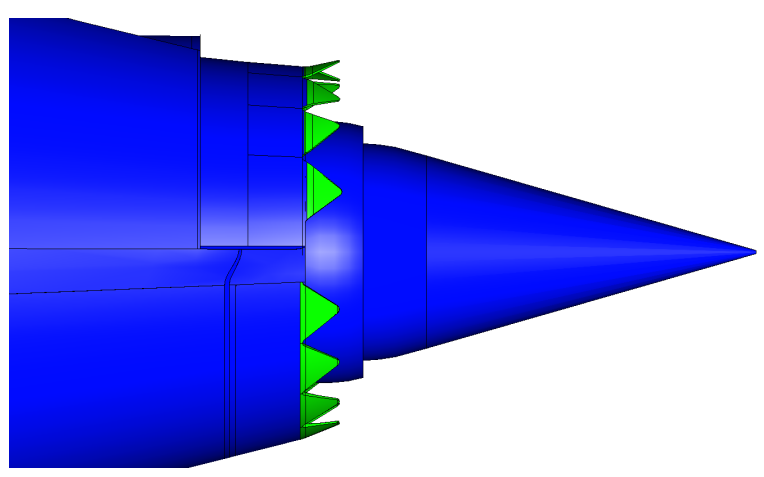

(d) 270 Configuration

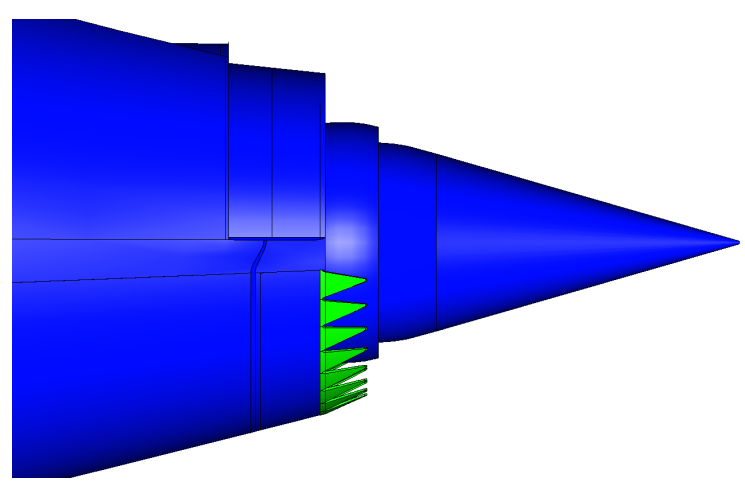

(e) 080 Configuration

Figure 3. Side views of the various chevron configurations. Test planform is not shown. 


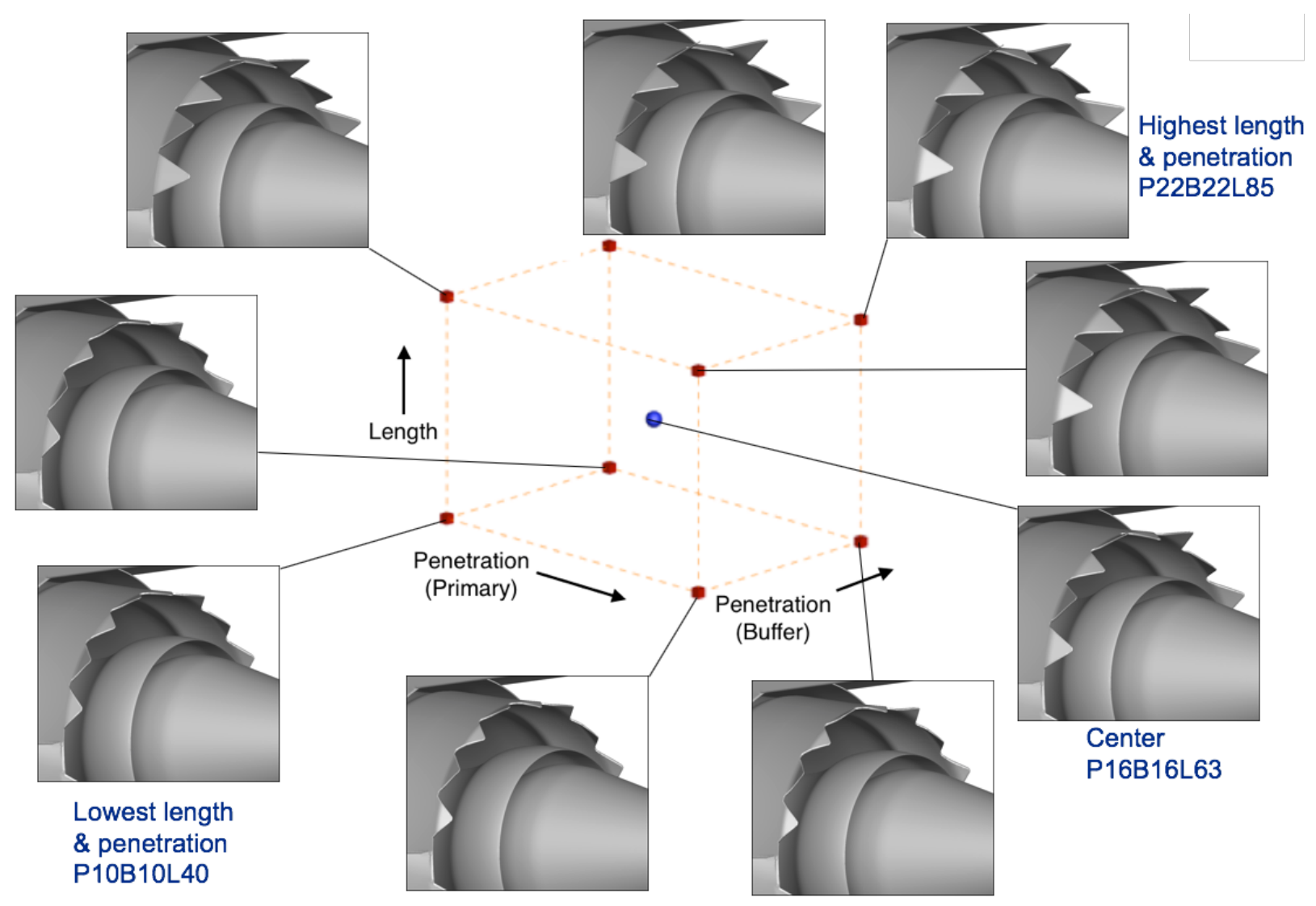

Figure 4. The MDOE parameter space. The PC2 chevrons are attached on the bottom of each nozzle.

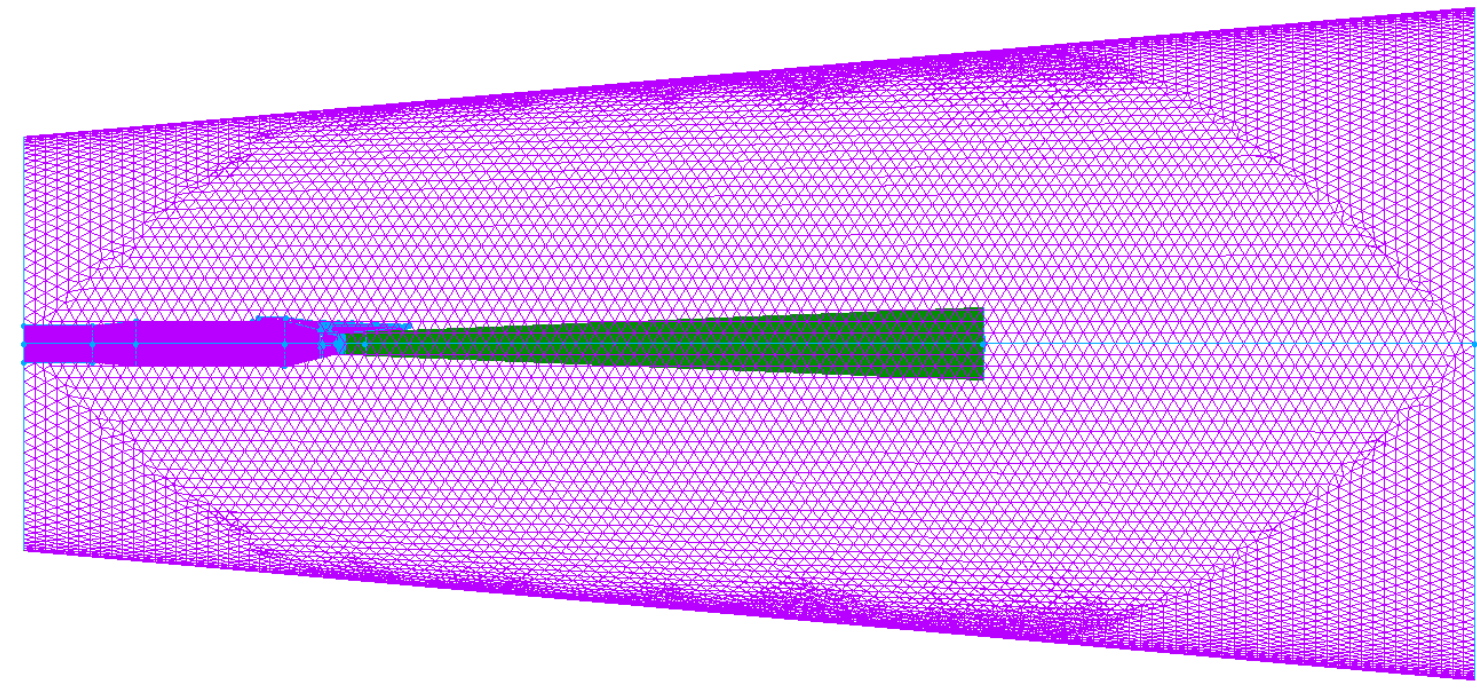

Figure 5. Installed nozzle grid domain. 


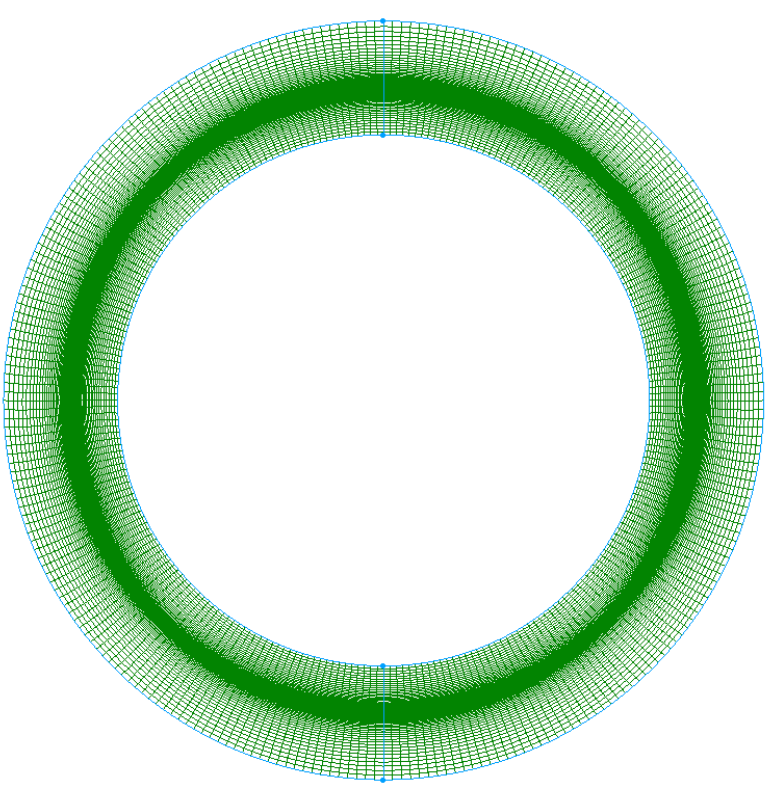

(a) $0.5 \mathrm{D}_{\mathrm{J}}$ downstream of primary nozzle exit.

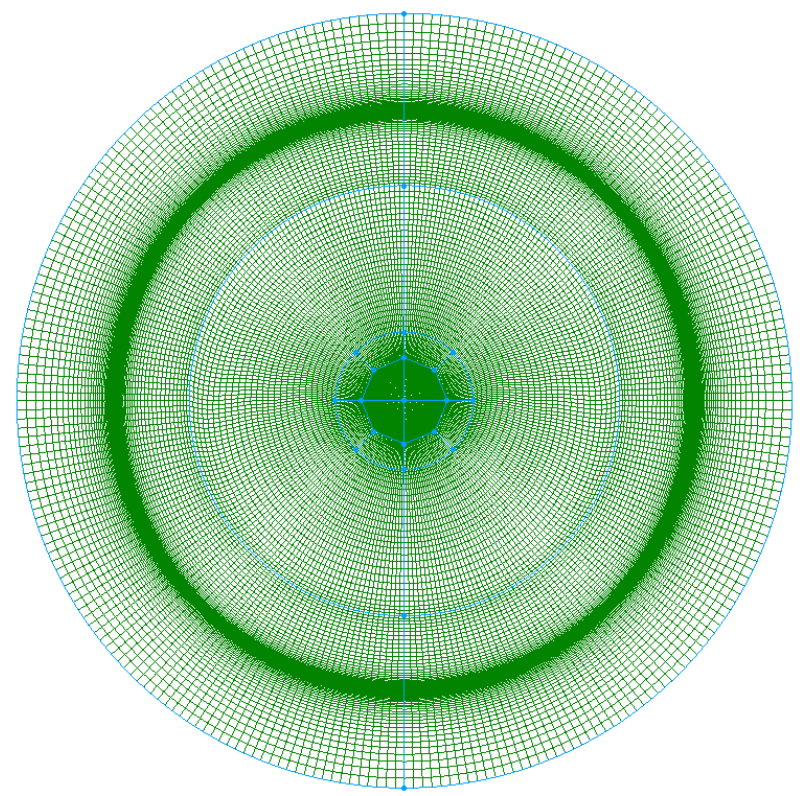

(b) $1.5 \mathrm{D}_{\mathrm{J}}$ downstream of primary nozzle exit.

Figure 6. Cross-sectional views of the hexahedral plume blocks.

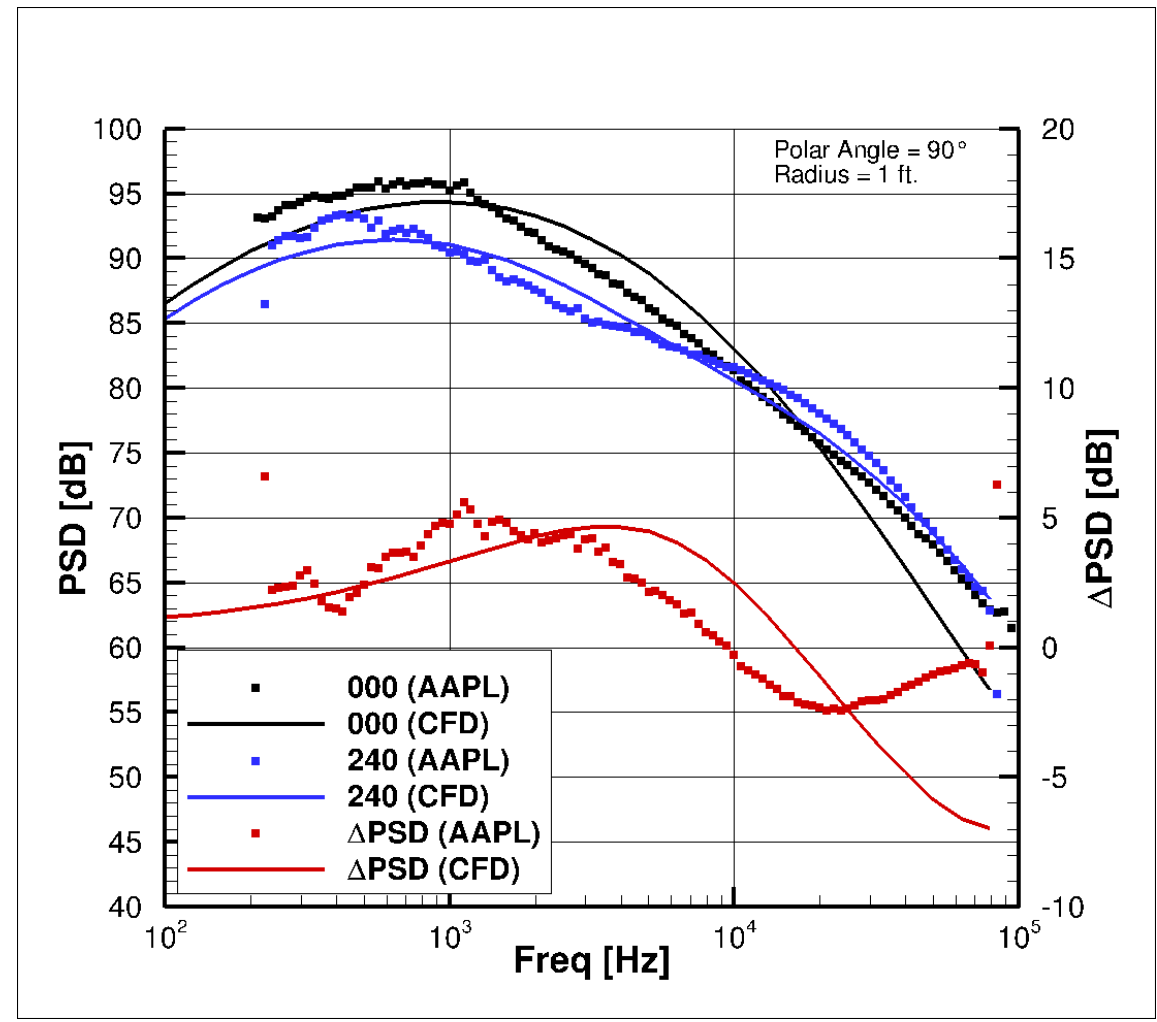

Figure 7. Comparison between CFD and test data of power spectral density for the baseline nozzle (000) and a chevron configuration (240). 
$\begin{array}{lll}\mathrm{x} / \mathrm{D}_{\mathrm{J}} & 000(\mathrm{PIV}) \quad 000(\mathrm{CFD}) \quad 240 \text { (PIV) } & 240 \text { (CFD) }\end{array}$

0.4
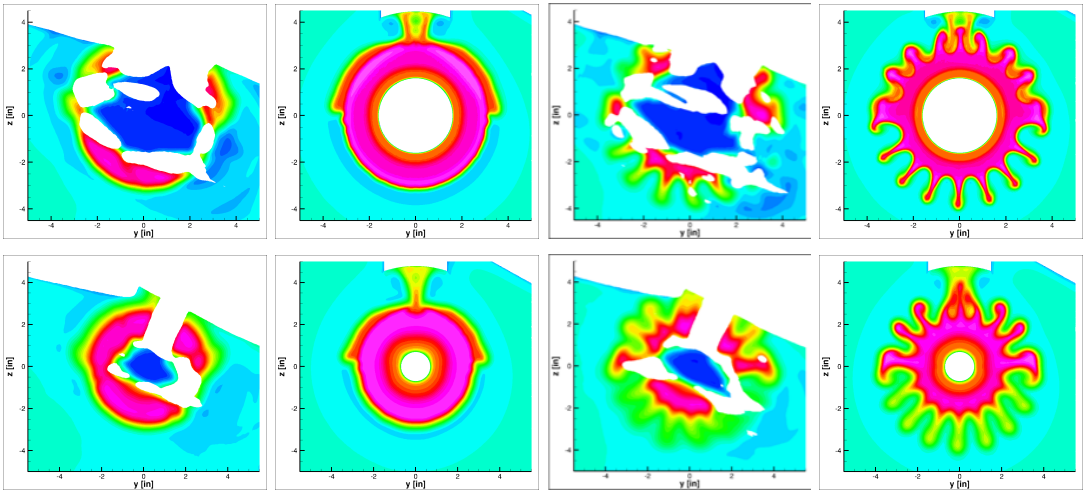

$\mathrm{U}[\mathrm{ft} / \mathrm{s}]$

0.9
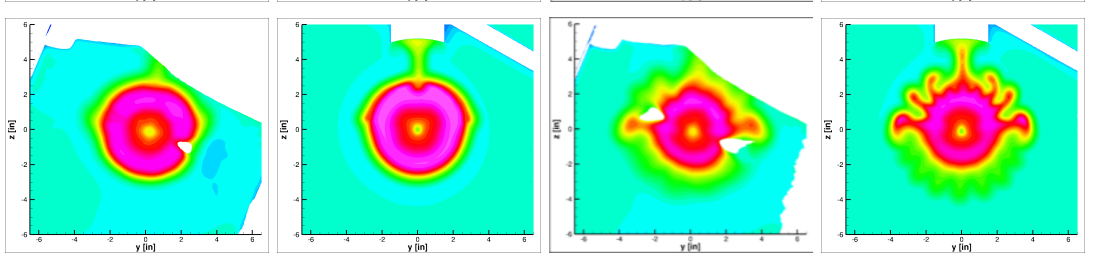

1600

1400

1200

1000

800

600

400

1.5
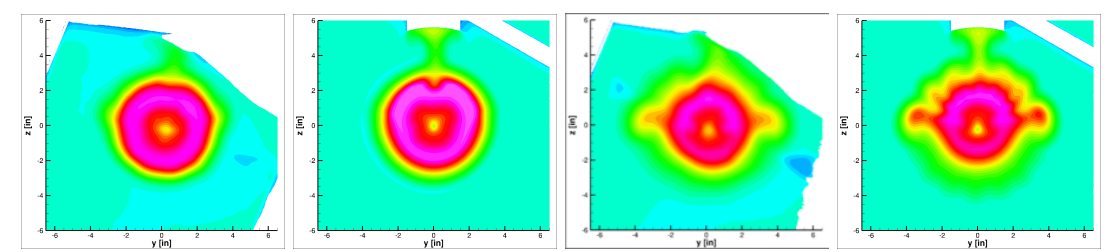

200

2.1
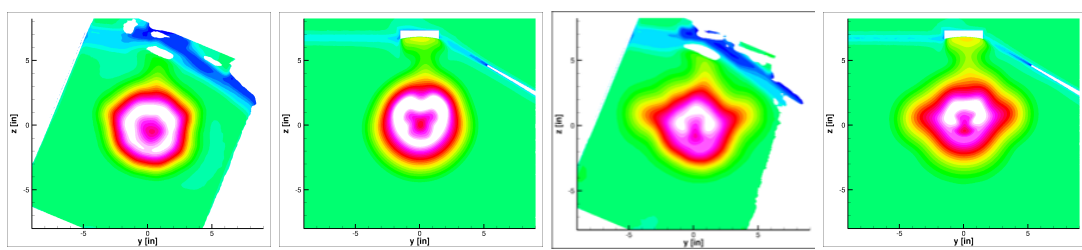

3.8
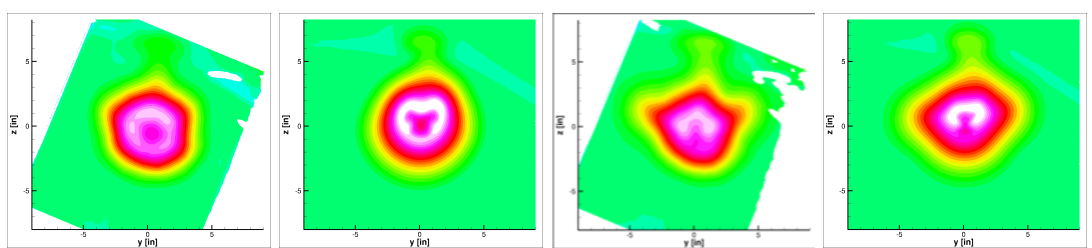

$\mathrm{U}[\mathrm{ft} / \mathrm{s}]$

5.0
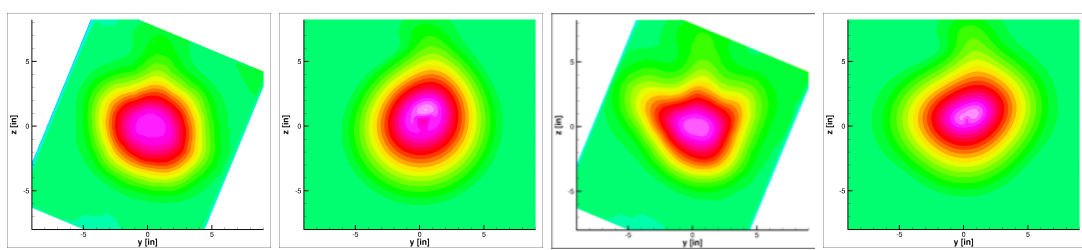

1200

1000

800

600

400

7.6
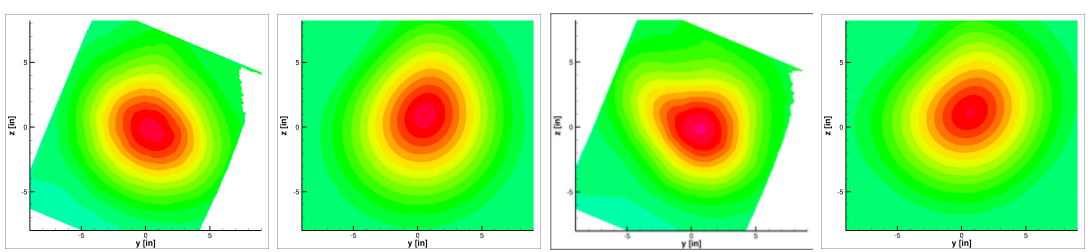

Figure 8. Comparison between PIV and CFD axial velocity for the base nozzle (000) and a chevron configuration (240). Axial location is relative to the primary stream exit. PIV data is blanked where quality is less than 0.8 . 
$\mathrm{x} / \mathrm{D}_{\mathrm{J}}$
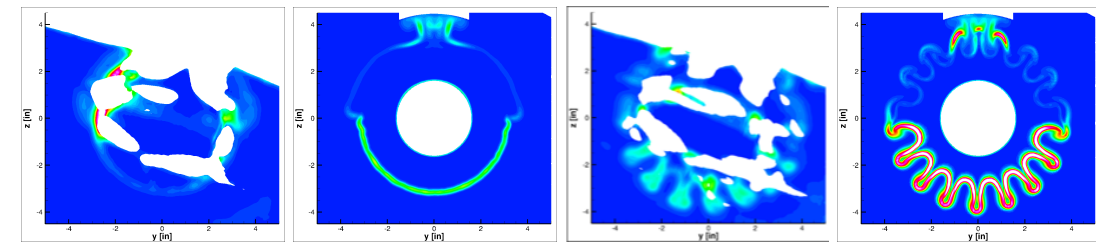

0.9
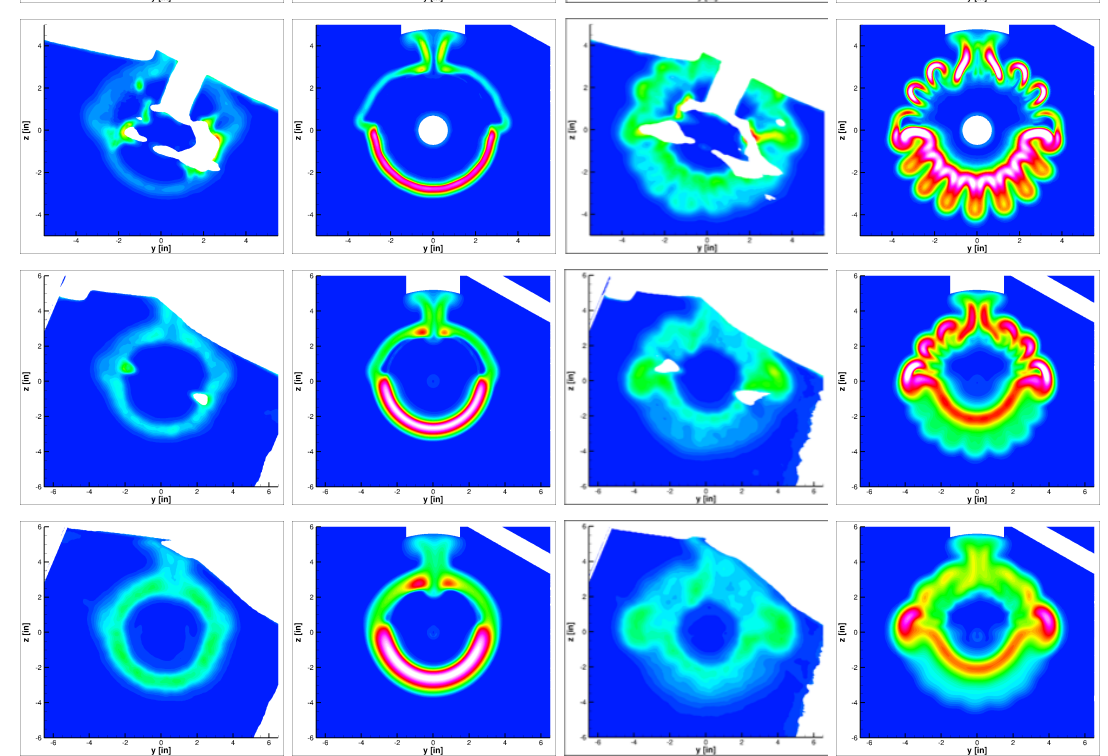

2.1
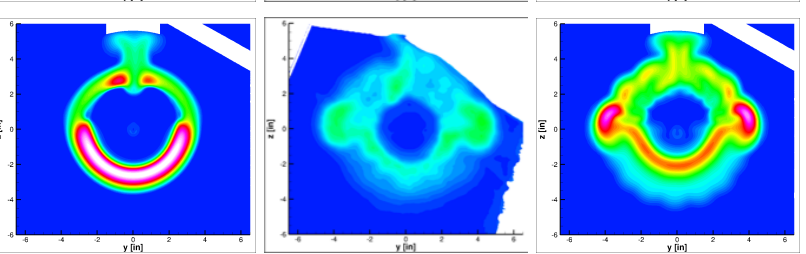

$\mathrm{k}\left[\mathrm{ft}^{\wedge} 2 / \mathrm{s}^{\wedge} 2\right]$
50000

45000

40000

35000

30000

25000
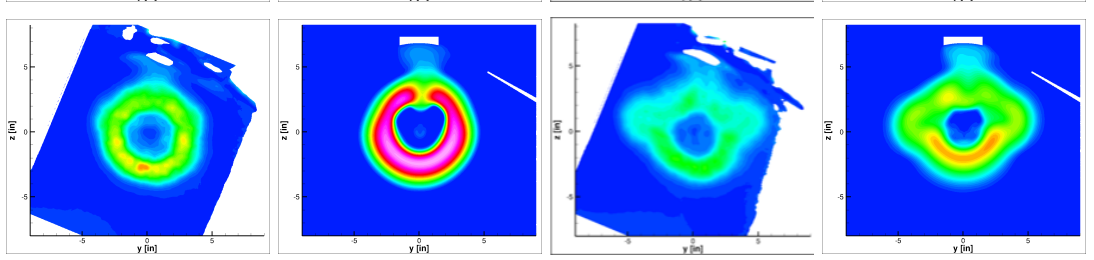

20000

15000

10000

3.8
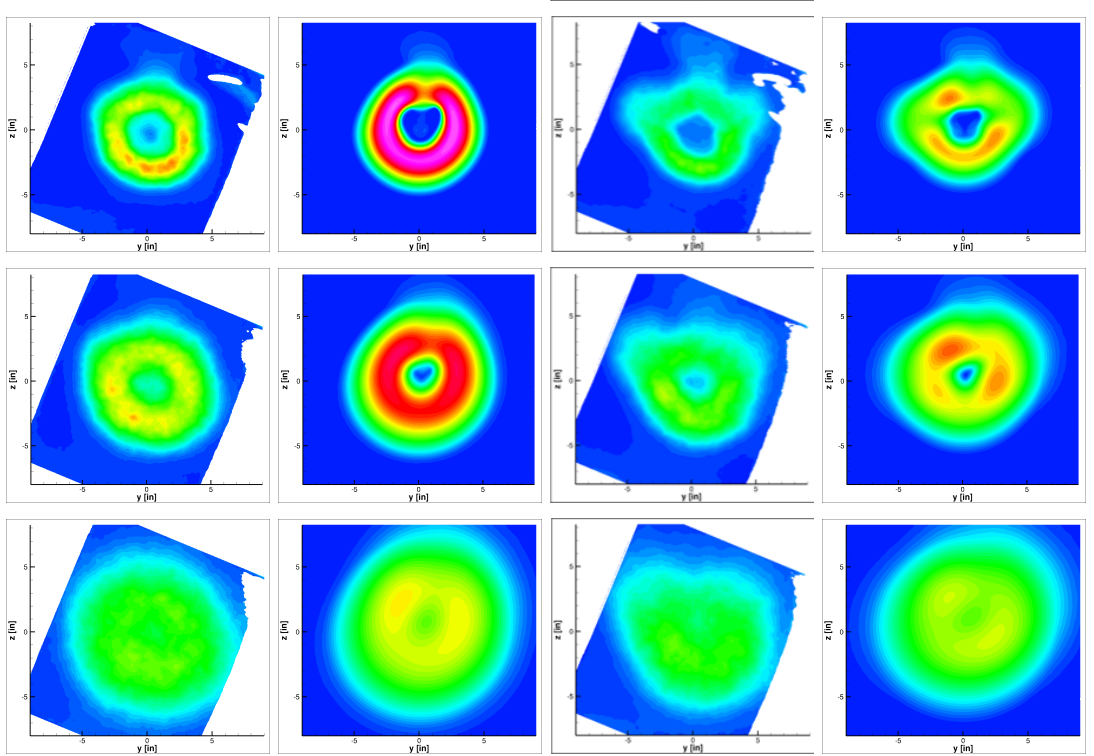

Figure 9. Comparison between PIV and CFD turbulent kinetic energy for the base nozzle (000) and a chevron configuration (240). Axial location is relative to the primary stream exit. PIV data is blanked where quality is less than 0.8 . 


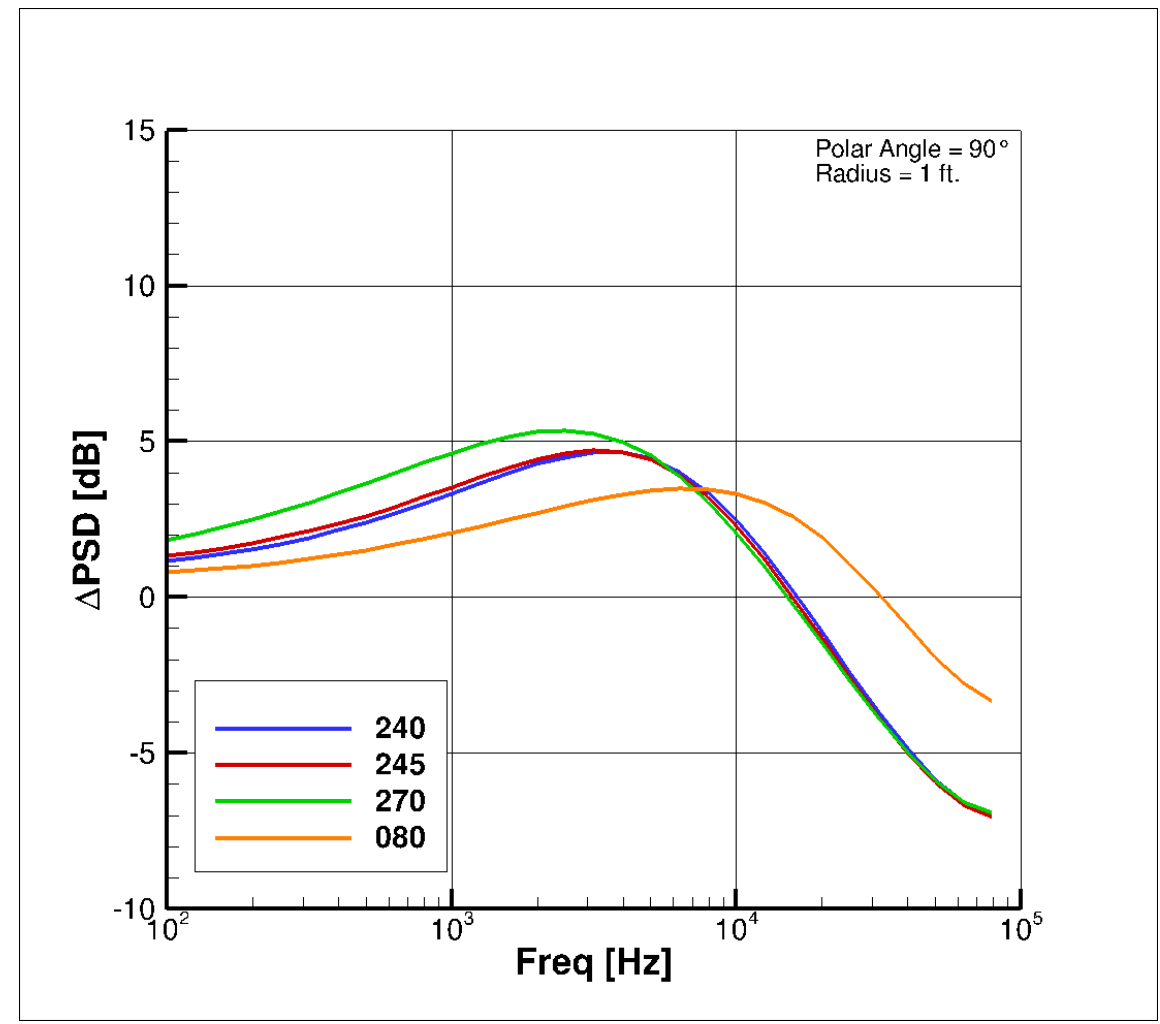

Figure 10. Power spectral density computed by $\mathrm{mSrc}$ for each chevron configuration. Numbers are relative to the baseline nozzle, where a positive value indicates a benefit from chevrons and a negative value designates a penalty. 
$\mathrm{x} / \mathrm{D}_{\mathrm{J}}$

0.4

0.9

1.5

2.1

3.8
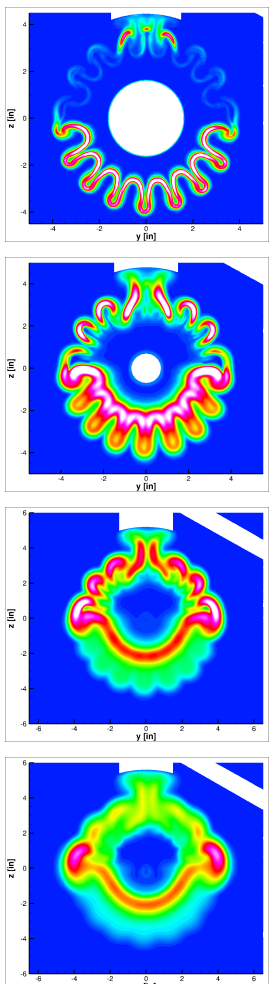

y
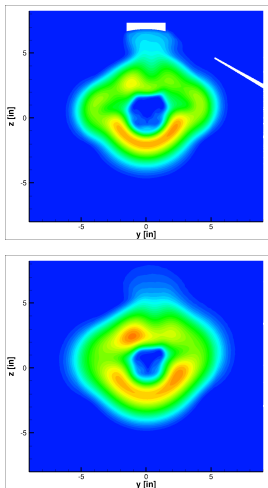

5.0

7.6

11.8

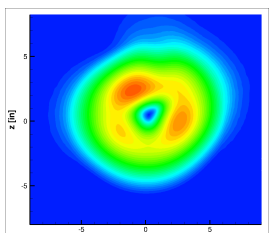

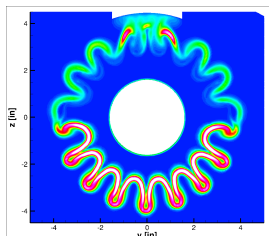
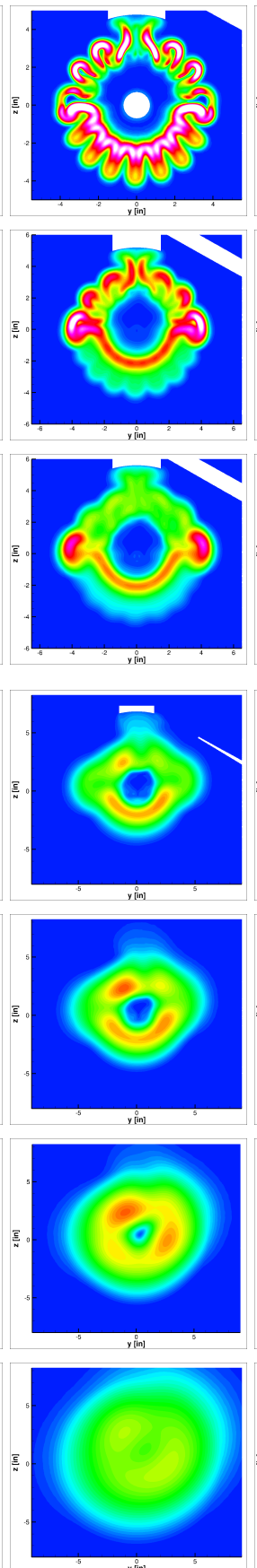

270
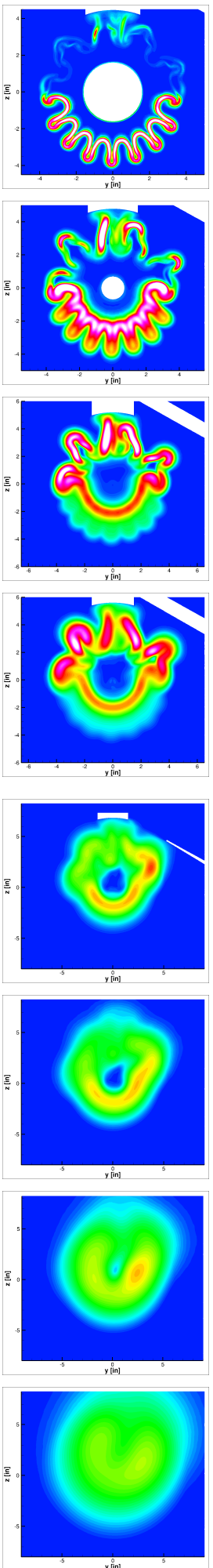

080
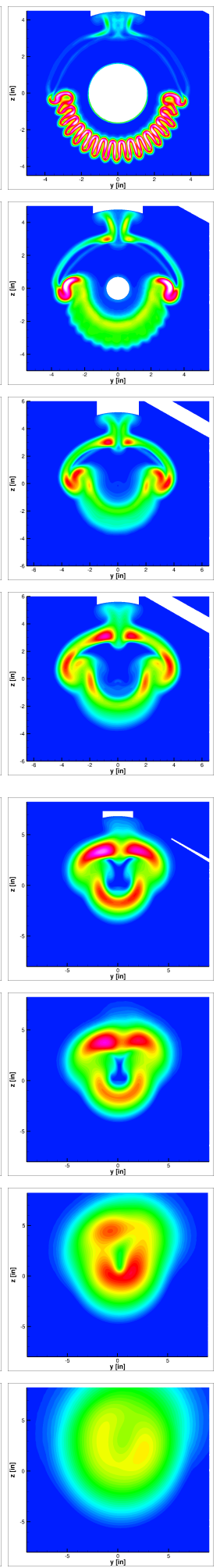

Figure 11. Comparison between turbulent kinetic energy for several chevron configurations. Axial location is relative to the primary stream exit. 
$\mathrm{x} / \mathrm{D}_{\mathrm{J}}$

0.4
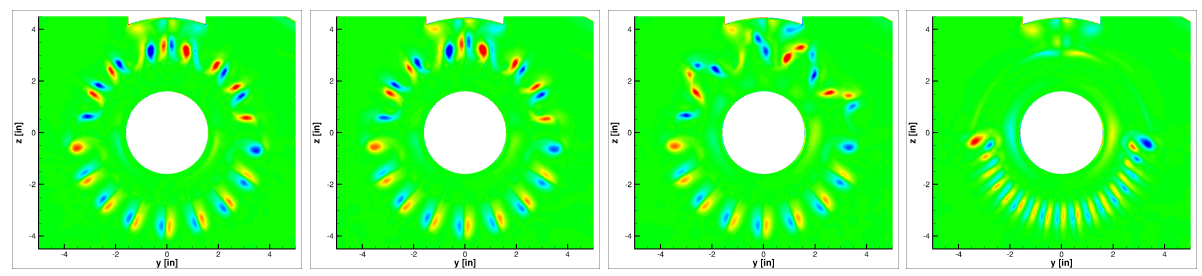

Vort_X [1/s]
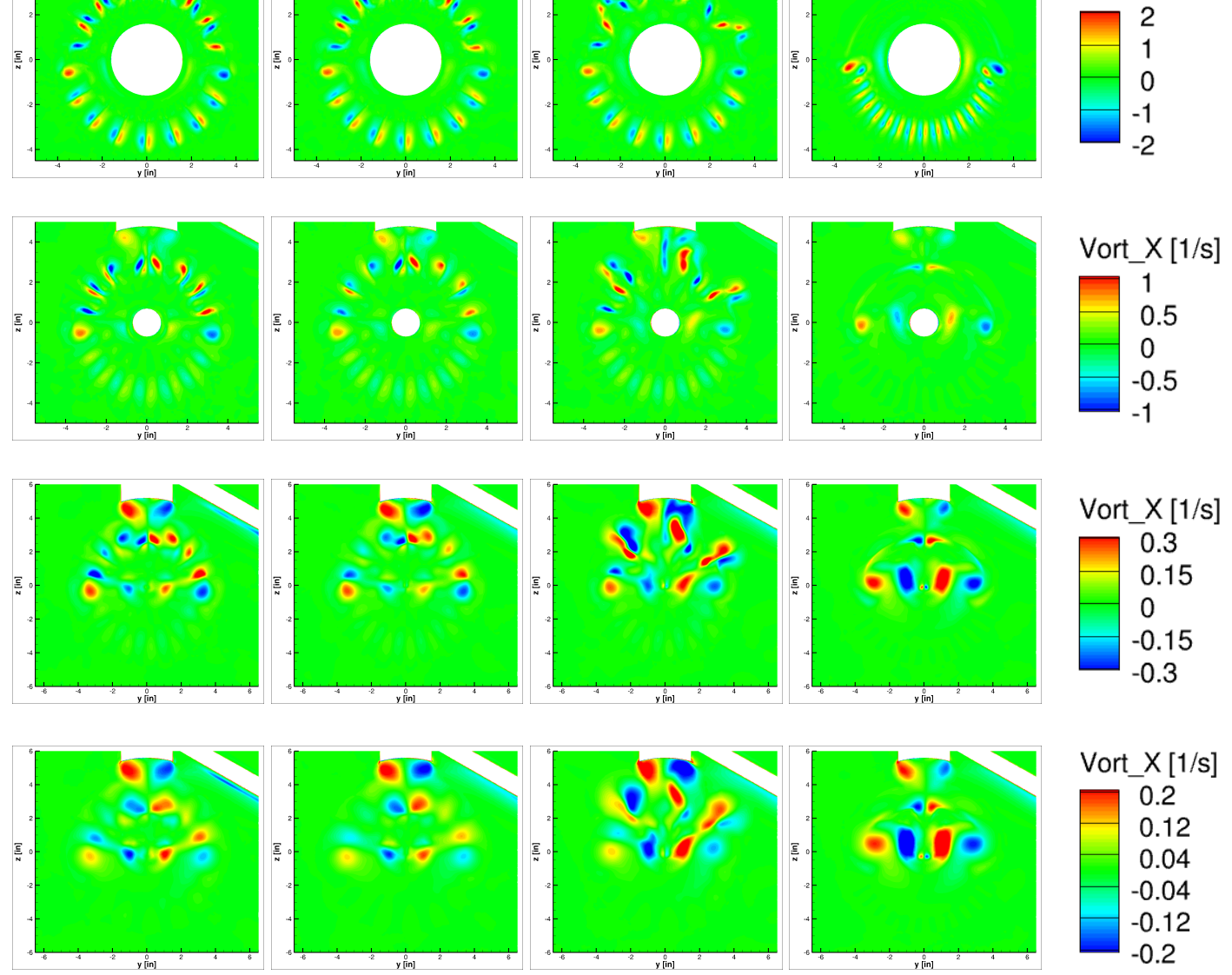

Figure 12. Comparison of axial vorticity for several chevron configurations. Axial location is relative to the primary stream exit. 


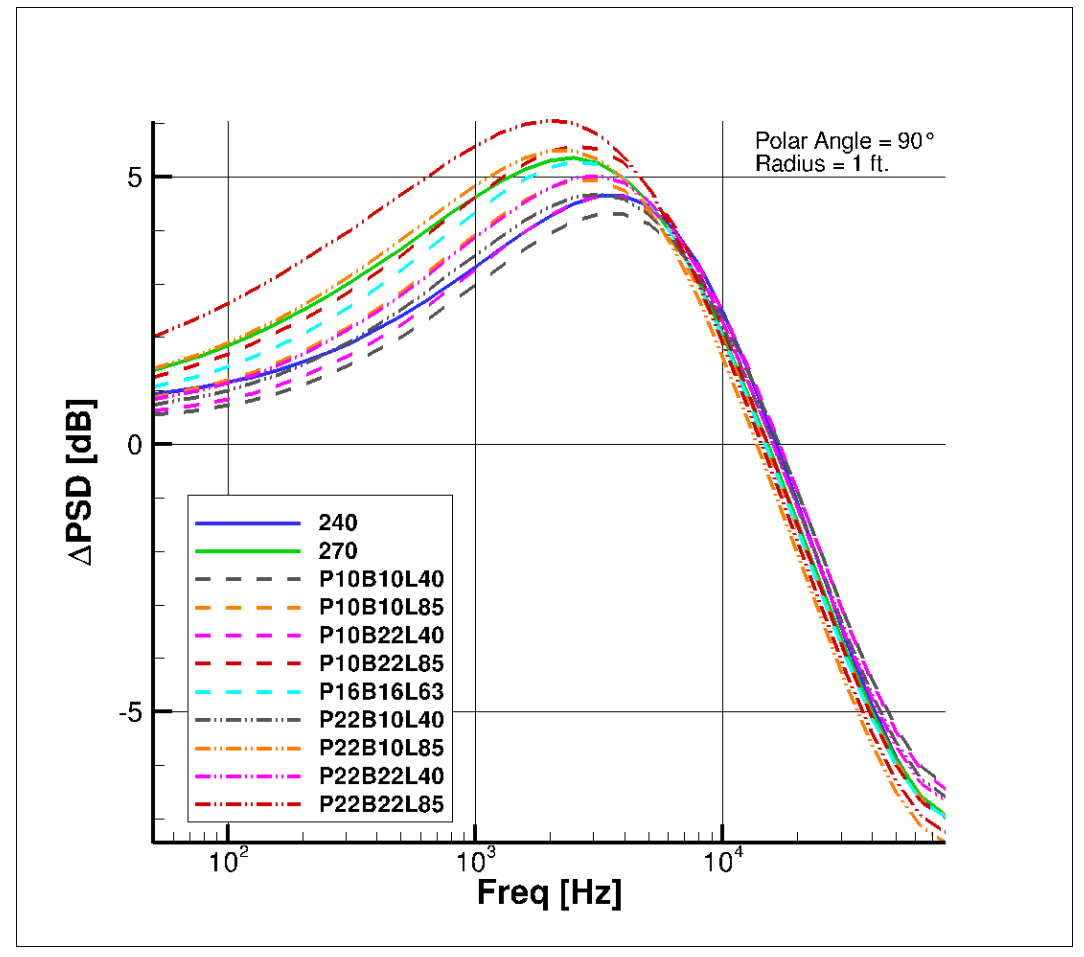

Figure 13. Comparison of power spectral density for each MDOE configuration.

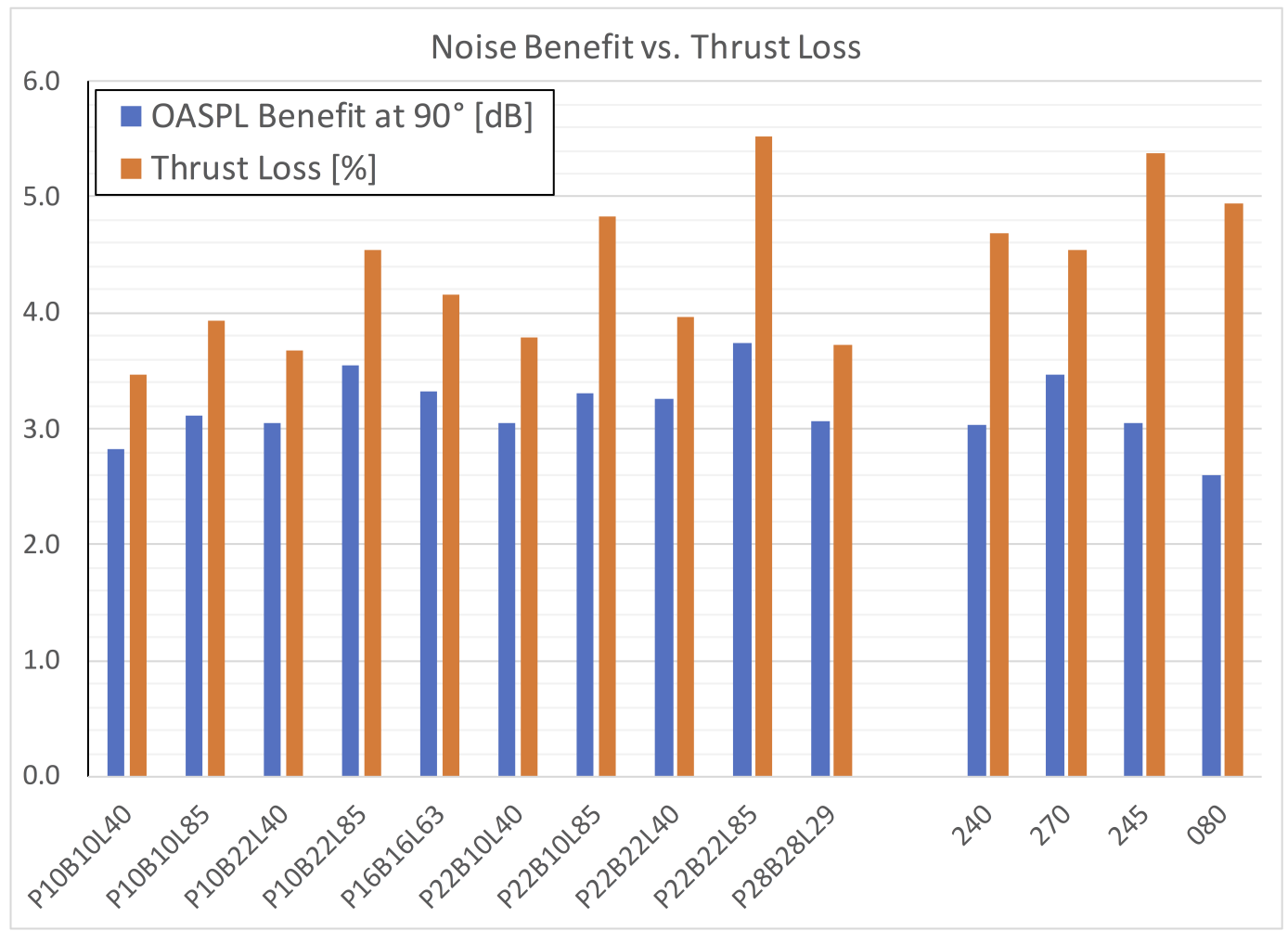

Figure 14. OASPL at $90^{\circ}$ and thrust loss for each chevron configuration. 